\title{
Estimated prediagnosis radiological progression: an important tool for studying the effects of early disease modifying antirheumatic drug treatment in rheumatoid arthritis
}

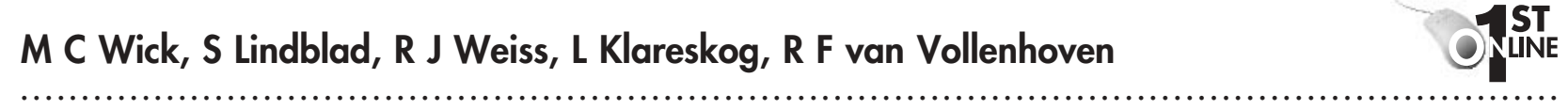

Objective: To determine if intrapatient comparisons between prediagnosis and subsequent radiological progression could be used to assess effects of DMARDs in an RA inception cohort.

Patients and methods: 149 non-randomised patients with newly diagnosed RA in four groups were analysed: patients treated with (a) methotrexate $(n=56)$; (b) sulfasalazine $(n=55)$; (c) auranofin $(n=19)$; and (d) controls who were poor treatment responders $(n=19)$. Radiographs were quantified using the Larsen erosion score. The prediagnosis radiological progression from the onset of RA symptoms to diagnosis was calculated and compared with the observed progression rate during the first year after diagnosis while receiving DMARD treatment.

Results: Mean (SD) disease duration from onset of symptoms until diagnosis was 6.7 (4.0) months. Mean (SD) baseline Larsen score was 13.2 (9.3), giving a mean (SD) estimated prediagnosis progression rate of 23.6 (12.4) Larsen score units/year. Control and auranofin groups showed radiological progression after diagnosis similar to the progression predicted by prediagnosis progression rates. Patients receiving methotrexate or sulfasalazine showed a marked reduction $(71 \%$ and $73 \%$, respectively; $p<0.001)$ in radiographic progression compared with prediagnosis progression. Conclusions: Prediagnosis rates of radiological progression can be used quantitatively to obtain information on the potential efficacy of DMARDs, and indicate that methotrexate and sulfasalazine, but not auranofin, significantly retard radiographic damage in the first year after diagnosis.

$\mathrm{R}$ adiological damage is an important outcome measure in rheumatoid arthritis (RA) ${ }^{1}$ and correlates well with functional outcome. ${ }^{2}$ It has been suggested that estimates of yearly progression of radiological damage, obtained by dividing changes in $x$ ray scores by time, can reliably be used for numerical comparisons between different treatments. ${ }^{3}$

Although current recommendations favour aggressive disease modifying antirheumatic drug (DMARD) treatment for early RA to prevent or slow radiological damage, recent studies have indicated that early DMARD treatment does not completely prevent radiographic progression even in the first year after diagnosis of RA. ${ }^{4}$ Thus, an important question is whether early DMARD treatment changes the rate of radiological progression. We investigated this question by assuming that radiographic damage begins at the onset of RA symptoms, making it possible to calculate the rate of untreated radiographic progression before diagnosis, and compare this rate with the observed rate of progression during the first year after diagnosis, during which period the patients were given DMARD treatment.

This study aimed at analysing the feasibility of this new method and using it to determine if the most commonly used DMARDs in our longitudinal early RA inception cohort reduced radiographic progression. Our data show that prediagnosis radiological progression can successfully be used in this manner, and suggest that methotrexate (MTX) and sulfasalazine (SSZ), but not auranofin (AUR), retard radiological progression in the first year of RA.

\section{PATIENTS AND METHODS}

We evaluated clinical data and radiographs of 149 patients with early RA registered in the Swedish Rheumatoid Arthritis Registry. Although the Swedish Rheumatoid Arthritis Registry is a collaborative effort of many Swedish rheumatology units, the data presented herein are based on patients treated in our unit at the Karolinska Hospital. Eligibility for inclusion in this study required that patients (a) were diagnosed with RA; $(b)$ evaluated clinically within the first year after onset of symptoms; (c) started DMARD treatment within 2 months after their first presentation to our early arthritis clinic; $(d)$ underwent at least 1 year of follow up to document laboratory and clinical values; $(e)$ had at least two sets of radiographs of hands and feet taken at baseline and during follow up, with the interval between the two sets of $x$ ray pictures not exceeding 1 year; and $(f)$ were treated with MTX $(n=56)$, SSZ $(n=55)$, or oral gold (AUR, $n=19)$, or, as a control group, we included patients who, because of poor clinical responses, had changed treatment at least twice during the first 2 years after diagnosis and whom we had previously shown ${ }^{6}$ to represent a group of poor treatment responders with persistent clinical activity (control, $n=19$ ). DMARDs were generally given as follows: AUR at $6 \mathrm{mg} /$ day; MTX at 7.5-15 mg/week; SSZ at $2 \mathrm{~g} /$ day.

\section{Measurements}

The following clinical measures were determined at baseline, and 3, 6, 9, and 12 months after diagnosis: Erythrocyte sedimentation rate (ESR), $\mathrm{C}$ reactive protein (CRP), American College of Rheumatology 28 tender and swollen joint counts (TJC, SJC), patient's global assessment $(0-10 \mathrm{~cm}$

Abbreviations: AUR, auranofin; CRP, C reactive protein; DAS28, 28 joint Disease Activity Score; DMARD, disease modifying antirheumatic drug; ESR, erythrocyte sedimentation rate; $H A Q$, Health Assessment Questionnaire; MTX, methotrexate; RA, rheumatoid arthritis; SJC, swollen joint count; SSZ, sulfasalazine; TJC, tender joint count; VAS, visual analogue scale 
Table 1 Baseline characteristics and values after 1 year of treatment in patients with early RA

\begin{tabular}{|c|c|c|c|c|c|}
\hline Treatment & $\begin{array}{l}\text { MTX } \\
(n=56)\end{array}$ & $\begin{array}{l}\text { SSZ } \\
(n=55)\end{array}$ & $\begin{array}{l}\text { AUR } \\
(n=19)\end{array}$ & $\begin{array}{l}\text { Control } \\
(n=19)\end{array}$ & $p^{*}$ Value \\
\hline \multicolumn{6}{|l|}{ Demographic characteristics } \\
\hline Age (years) & $63.4(13.0)$ & $53.6(15.9)$ & $59.1(13.1)$ & $50.2(16.3)$ & 0.001 \\
\hline Men, No (\%) & $16(29)$ & $24(44)$ & $7(37)$ & $4(21)$ & \\
\hline Women, No (\%) & 40 (71) & $31(56)$ & $12(63)$ & $15(79)$ & \\
\hline Disease duration (months) & $6.8(3.9)$ & $6.5(3.6)$ & $7.0(4.4)$ & $6.5(4.2)$ & 0.8 \\
\hline \multicolumn{6}{|l|}{ Clinical baseline values } \\
\hline ESR $(\mathrm{mm} / 1 \mathrm{st} h)$ & $29.5(20.3)$ & $28.2(23.1)$ & $16.3(9.6)$ & $29.9(21.9)$ & 0.1 \\
\hline $\mathrm{CRP}(\mathrm{mg} / \mathrm{l})$ & $32(40)$ & $19(18)$ & $11(9)$ & $29(32)$ & 0.03 \\
\hline DAS28 & $5.4(1.1)$ & $4.8(1.1)$ & $4.1(0.9)$ & $5.0(1.0)$ & 0.001 \\
\hline $\mathrm{HAQ}$ & $1.1(0.6)$ & $0.8(0.5)$ & $0.8(0.5)$ & $1.1(0.6)$ & 0.01 \\
\hline †SJC & $11.0(5.6)$ & $10.3(5.8)$ & $4.5(3.5)$ & $7.6(5.4)$ & 0.001 \\
\hline †TJC & $9.8(6.2)$ & $7.5(6.0)$ & $4.6(3.7)$ & $7.2(5.5)$ & 0.008 \\
\hline †Pain & $4.8(2.3)$ & $3.7(2.1)$ & $3.9(2.5)$ & $5.3(2.6)$ & 0.02 \\
\hline$\ddagger$ Patient's assessment & $4.5(2.4)$ & $3.7(2.5)$ & $4.4(2.4)$ & $5.6(2.7)$ & 0.1 \\
\hline Larsen score & $15.6(9.6)$ & $12.9(9.4)$ & $9.6(7.0)$ & $9.7(7.7)$ & 0.03 \\
\hline \multicolumn{6}{|c|}{ Clinical values after 1 year of treatment } \\
\hline $\mathrm{ESR}(\mathrm{mm} / 1 \mathrm{st} \mathrm{h})$ & $19.5(16.8)$ & $15.6(16.5)$ & $12.3(9.2)$ & $19.8(17.1)$ & 0.001 \\
\hline $\mathrm{CRP}(\mathrm{mg} / \mathrm{l})$ & $17(26)$ & $12(13)$ & $9(7)$ & $19(23)$ & 0.001 \\
\hline DAS28 & $3.8(1.5)$ & $3.2(1.5)$ & $3.1(1.2)$ & $4.2(1.2)$ & 0.001 \\
\hline $\mathrm{HAQ}$ & $0.8(0.5)$ & $0.5(0.5)$ & $0.7(0.5)$ & $1.0(0.6)$ & 0.001 \\
\hline$\dagger S J C$ & $4.8(5.4)$ & $4.0(5.0)$ & $2.3(2.9)$ & $5.0(4.6)$ & 0.001 \\
\hline †TJC & $4.6(5.3)$ & $3.5(4.8)$ & $2.7(3.5)$ & $5.5(4.9)$ & 0.001 \\
\hline †Pain & $3.6(2.4)$ & $2.8(2.4)$ & $3.1(2.2)$ & $4.4(2.5)$ & 0.001 \\
\hline$\ddagger$ Patient's assessment & $3.5(2.4)$ & $2.8(2.3)$ & $3.3(2.4)$ & $4.5(2.6)$ & 0.001 \\
\hline Larsen score & $23.2(11.3)$ & $18.9(12.6)$ & $18.9(9.8)$ & $20.0(8.5)$ & 0.2 \\
\hline
\end{tabular}

Results are shown as mean (SD) unless indicated otherwise.

*Analysis of variance followed by post hoc Bonferroni's test; tbased on a 28 joint count; festimated by (VAS 0$10 \mathrm{~cm}$.

visual analogue scale (VAS)), pain (0-10 cm VAS), Health Assessment Questionnaire (HAQ), and the Disease Activity Score DAS28.

\section{Radiographs}

Identical radiographs of the hands and feet were taken of all patients in posteroanterior and tangential views at baseline and at 1 year follow up. Radiographs were assessed after achieving satisfactory intraobserver reliability (0.93-0.95). The radiographs were scored blinded to treatment, in pairs (hands and feet), and in chronological sequence applying the Larsen method. ${ }^{8}$ The scoring procedure was performed and documented by an experienced investigator (MCW) using the computer assisted quantification software "X-Ray RheumaCoach". ${ }^{9}$ In each case, 32 joints were scored: 8 proximal interphalangeal, 2 thumb interphalangeal, 10 metacarpophalangeal, 2 wrists, 2 hallux interphalangeal, and 8 metatarsophalangeal joints (II-V). Each wrist was scored as a unit and multiplied by five.

The aggregate Larsen score was modified slightly by excluding grade 1 , so that the scale became $0-4$ (grades 1 and 0 were combined and designated grade 0 , grade 2 was called grade 1, grade 3 was called grade 2 , grade 4 was called grade 3, and grade 5 was called grade 4$).{ }^{10}$ Thus, the maximum possible score was 160 . The change in the Larsen score was calculated by subtracting baseline Larsen score values from the respective annual score. The rate of radiological progression over time was calculated, taking the first onset of RA symptoms as the initiation of prediagnosis radiographic destruction.

\section{Statistical analysis}

All data are given as means (SD). Statistical comparisons were performed using analyses of variance followed by post hoc Bonferroni's test and Student's $t$ test. All statistical analyses were performed using SPSS, version 11.5 for Windows (SPSS Inc, Chicago, IL).

\section{RESULTS}

Table 1 summarises the demographic, clinical, and radiological baseline values and 1 year follow up data of the 149 patients included in this analysis. Of note, this was not a randomised trial, because the choice of treatment was based on individual patient clinical considerations. Thus, these patients represented a "real life" sample of patients with early RA at our early arthritis centre. Differences between the

Table 2 Estimated rates of radiological progression before diagnosis (and start of DMARD treatment) and change in Larsen scores during year 1 (Larsen score units/year (SD))

\begin{tabular}{|c|c|c|c|c|c|}
\hline Treatment & $\begin{array}{l}\text { MTX } \\
(n=56)\end{array}$ & $\begin{array}{l}\text { SSZ } \\
(n=55)\end{array}$ & $\begin{array}{l}\text { AUR } \\
(n=19)\end{array}$ & $\begin{array}{l}\text { Control } \\
(n=19)\end{array}$ & $\mathrm{p}^{*}$ Value \\
\hline $\begin{array}{l}\text { Prediagnosis progression rate } \text { (change/year) } \\
\text { Progression from baseline to year } 1 \\
\text { Decrease in rate of progression }\end{array}$ & $\begin{array}{l}27.4(16.5) \\
7.9(4.6) \\
-19.5(9.3) \\
p<0.001 \ddagger\end{array}$ & $\begin{array}{l}23.8(16.9) \\
6.5(4.9) \\
-17.3(10.2) \\
p<0.001 \ddagger\end{array}$ & $\begin{array}{l}16.6(11.5) \\
9.4(6.8) \\
-7.2(4.4) \\
p=N S \ddagger\end{array}$ & $\begin{array}{l}17.6(13.6) \\
10.4(4.9) \\
-7.2(4.8) \\
p=\text { NSf }\end{array}$ & $\begin{array}{l}0.1 \\
0.025 \\
0.03 \\
-\end{array}$ \\
\hline
\end{tabular}

*Comparison of four groups by analysis of variance; the prediagnosis radiological progression rate was estimated by dividing the radiological damage at baseline by the symptom duration as recorded at the baseline visit for each patient; †comparison of prediagnosis progression with progression from baseline to year 1, for each group (paired Student's $t$ test). 

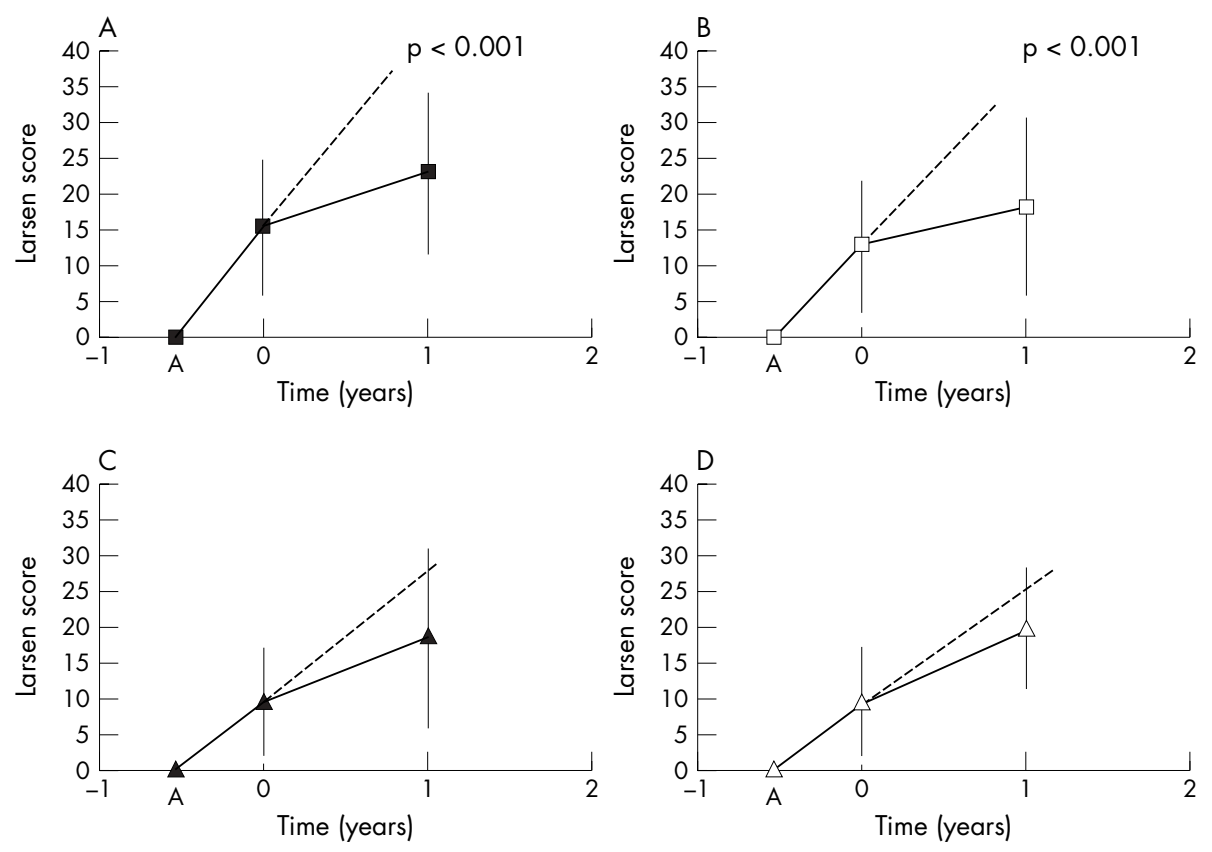

$$
\begin{aligned}
& A=\text { Onset of RA symptoms } \\
& \rightarrow-\text { MTX } \square-\text { SSZ } \\
& \triangle \text { AUR } \triangle \text { Control }
\end{aligned}
$$

Figure 1 Increment of Larsen score in the four different treatment groups: (A) MTX; (B) SSZ; (C) AUR; and (D) controls; means (SD) are shown. Prediagnosis radiological progression is represented as a straight line starting at the time of symptom onset. Estimated progression from baseline to year 1 is represented as a dotted line extending from the prediagnosis radiological progression. Actual observed radiological progression is represented as an unbroken line.

groups were seen for some baseline values (table 1). The mean age at RA diagnosis and institution of treatment was 57.5 (15.3) years. The mean time from onset of symptoms (as recorded by the patient) until diagnosis of RA was 6.7 (4.0) months. None of the patients received any DMARD treatment before diagnosis.

Clinical outcomes such as TJC, SJC, and the DAS28 improved from baseline to year 1 with DMARD treatment, but not in the control group (table 1). The mean baseline Larsen score was 13.2 (9.3) (median 12, interquartile range 5.8-18.5) and increased to 20.5 (10.8) (median 20, interquartile range 11.0-29.0) during 1 year of treatment-that is, the mean increase in radiographic damage from baseline to year 1 was 7.9 (6.0).

Taking the first onset of RA symptoms as the earliest possible start of radiological destruction, the radiological progression rate up to the time of diagnosis was calculated. For all 149 patient, the rate was 23.6 (12.4) Larsen score units/year and for the four groups the prediagnosis progression rate varied from 16.6 (11.5) to 27.4 (16.5) (table 2). When the estimated prediagnosis progression rate was compared with the progression occurring during year 1 after diagnosis (fig 1), the progression rate was found to be reduced in all groups. However, the reduction in radiographic progression rate in the MTX and SSZ groups was much greater and significant (reductions of $71 \%$ and $73 \%$, respectively; $\mathrm{p}<0.001$ either treatment when compared with predicted progression), whereas the reductions in radiographic progression rate were smaller and not significant in the AUR and control patient groups $(43 \%$ and $41 \%$, respectively) (table 2 ). The decreases in progression rate were significantly different between the four groups by analysis of variance $(\mathrm{p}<0.05)$.

\section{DISCUSSION}

We wished to use an important and, in some aspects, new method of comparing radiological progression before diagnosis with progression seen during the first year after diagnosis in order to study the effects of early intervention with DMARDs. The method in this study should not to be confused with the recently described method of estimating radiological progression before inclusion in a clinical trial in patients with longstanding RA. ${ }^{11}$

The results presented herein demonstrate the feasibility of this approach and suggest that administration of MTX or SSZ, but not AUR, significantly attenuates the destructive course of this disease already in the first year after diagnosis.

Recent emphasis on early intervention complicates identification of appropriate control groups in longitudinal studies designed to evaluate various therapeutic regimens. The control group included in this study was defined as patients with RA who were considered to be "poor clinical responders", and who underwent changes in DMARD treatment at least twice during the first 2 years after their initial diagnosis. During follow up these patients showed no perceptible improvement in clinical disease activity indices, making them a useful control group for comparisons in a longitudinal database, and indeed, compared with MTX, SSZ, and AUR, control patients showed the greatest increase of radiographic score from baseline to year 1 . The numbers of patients in the AUR and control groups were relatively small for a long term observational study, limiting the generalisability of our results.

We used the time of onset of RA symptoms as the earliest initiation of radiological destruction to estimate prediagnosis progression rates. Hypothetically, the first radiological damage might have preceded the earliest reported symptoms, 
but then the data would suggest that radiographic progression increased after the institution of DMARD treatment, which is less likely. Conversely, it is also possible that radiological progression starts after the first clinical symptoms become apparent. In this case, our results suggest even more convincingly that early intervention with MTX and SSZ retards radiological damage compared with AUR treated or non-responder controls. It needs to be mentioned, however, that the estimate of prediagnosis radiological progression in patients with very short duration of symptoms may sometimes be an overestimate.

In conclusion, estimates of prediagnosis radiographic progression can be used quantitatively to assess the potential therapeutic efficacy of DMARDs in early RA, and suggest that both MTX and SSZ, but not AUR, significantly retard radiographic damage in the first year after diagnosis. Prediagnosis rates of radiological progression can be an important tool for assessing the benefit of early, aggressive treatment in RA.

\section{Authors' affiliations}

M C Wick, S Lindblad, L Klareskog, R F van Vollenhoven,

Rheumatology Unit, Department of Medicine at Karolinska Hospital, Stockholm, Sweden

R J Weiss, Department of Orthopaedic Surgery, Karolinska Hospital, Stockholm, Sweden

Correspondence to: Dr M C Wick, Department of Radiology, Innsbruck Medical University, Anichstrasse 35, A-6020 Innsbruck, Austria; marius.wick@uibk.ac.at

Accepted 26 March 2004

Published Online First 19 April 2004

\section{REFERENCES}

1 van der Heijde DM, Boers M, Lassere M. Methodological issues in radiographic scoring methods in rheumatoid arthritis. J Rheumatol 1999;26:726-30.

2 Scott DL, Pugner K, Kaarela K, Doyle DV, Woolf A, Holmes J, et al. The links between joint damage and disability in rheumatoid arthritis. Rheumatology (Oxford) 2000;39:122-32.

3 Strand V, Landewe R, van der Heijde D. Using estimated yearly progression rates to compare radiographic data across recent randomised controlled trials in rheumatoid arthritis. Ann Rheum Dis 2002;61(suppl II):ii64-6.

4 Mulherin D, Fitzgerald O, Bresnihan B. Clinical improvement and radiological deterioration in rheumatoid arthritis: evidence that the pathogenesis of synovial inflammation and articular erosion may differ. $\mathrm{Br} J$ Rheumatol 1997;35:1263-8.

5 van der Horst-Bruinsma IE, Speyer I, Visser H, Breedveld FC, Hazes JM Diagnosis and course of early-onset arthritis: results of a special early arthritis clinic compared to routine patient care. $\mathrm{Br} J$ Rheumatol 1998;37:1084-8.

6 Wick MC, Lindblad S, Klareskog L, van Vollenhoven R. Radiological progression despite clinical efficacy of DMARD-treated RA in an inception cohort: a retrospective comparison of four treatments [abstract]. Ann Rheum Dis 2003;62(suppl I): 154 .

7 van der Heijde DM, van't Hof MA, van Riel PL, Theunisse LA, Lubberts EW, van Leeuwen MA, et al. Judging disease activity in clinical practice in rheumatoid arthritis: first step in the development of a disease activity score. Ann Rheum Dis 1991;49:916-20.

8 Larsen A, Dale K, Eek M. Radiographic evaluation of rheumatoid arthritis and related conditions by standard reference films. Acta Radiol Diagn (Stockh) 1977; 18:481-91.

9 Wick M, Peloschek P, Bögl K, Graninger W, Smolen JS, Kainberger F. The "X-Ray RheumaCoach" software: a novel tool for enhancing the efficacy and accelerating radiological quantification in rheumatoid arthritis. Ann Rheum Dis 2003:62:579-82

10 Plant MJ, Williams AL, O'Sullivan MM, Lewis PA, Coles EC, Jessop JD. Relationship between time-integrated $C$-reactive protein levels and radiologic progression in patients with rheumatoid arthritis. Arthritis Rheum 2000:43:1473-7.

11 Strand V, Sharp JT. Radiographic data from recent randomized controlled trials in rheumatoid arthritis: what have we learned? Arthritis Rheum 2003;48:21-34. 